OPEN ACCESS

Edited by:

Cicero Matthew R. Habito,

Massachusetts General

Hospital, USA

Reviewed by:

Goutam Chakraborty,

Memorial Sloan Kettering Cancer

Center, USA

Johanna Patricia Adevoso Canal,

University of the Philippines

Manila, Philippines

*Correspondence:

Marie-France Penet

mpenet@mri.jhu.edu

Specialty section:

This article was submitted to Cancer Imaging and Diagnosis, a section of the journal

Frontiers in Oncology

Received: 22 March 2016 Accepted: 02 November 2016 Published: 16 November 2016

Citation:

Penet MF, Krishnamachary B, Wildes F, Mironchik Y,

Mezzanzanica D, Podo F,

de Reggi M, Gharib B and

Bhujwalla ZM (2016) Effect of

Pantethine on Ovarian Tumor Progression and Choline Metabolism.

Front. Oncol. 6:244.

doi: 10.3389/fonc.2016.00244

\section{Effect of Pantethine on Ovarian Tumor Progression and Choline Metabolism}

\author{
Marie-France Penet ${ }^{1,2 *}$, Balaji Krishnamachary ${ }^{1}$, Flonne Wildes ${ }^{1}$, Yelena Mironchik', \\ Delia Mezzanzanica ${ }^{3}$, Franca Podo 4 , Max de Reggi ${ }^{5}$, Bouchra Gharib ${ }^{5}$ and \\ Zaver M. Bhujwalla ${ }^{1,2}$
}

' JHU ICMIC Program, Russell H. Morgan, Division of Cancer Imaging Research, Department of Radiology and Radiological Science, The Johns Hopkins University School of Medicine, Baltimore, MD, USA, ${ }^{2}$ Sidney Kimmel Comprehensive Cancer Center, The Johns Hopkins University School of Medicine, Baltimore, MD, USA, ${ }^{3}$ Unit of Molecular Therapies, Department of Experimental Oncology and Molecular Medicine, Fondazione IRCCS Istituto Nazionale dei Tumori, Milan, Italy, ${ }^{4}$ Section of Molecular and Cellular Imaging, Department of Cell Biology and Neurosciences, Istituto Superiore di Sanità, Rome, Italy,

${ }^{5}$ Neurobiology of Cellular Interactions and Neurophysiopathology (NICN), Aix Marseille Univ, CNRS, Marseille, France

Epithelial ovarian cancer remains the leading cause of death from gynecologic malignancy among women in developed countries. New therapeutic strategies evaluated with relevant preclinical models are urgently needed to improve survival rates. Here, we have assessed the effect of pantethine on tumor growth and metabolism using magnetic resonance imaging and high-resolution proton magnetic resonance spectroscopy (MRS) in a model of ovarian cancer. To evaluate treatment strategies, it is important to use models that closely mimic tumor growth in humans. Therefore, we used an orthotopic model of ovarian cancer where a piece of tumor tissue, derived from an ovarian tumor xenograft, is engrafted directly onto the ovary of female mice, to maintain the tumor physiological environment. Treatment with pantethine, the precursor of vitamin B5 and active moiety of coenzyme A, was started when tumors were $\sim 100 \mathrm{~mm}^{3}$ and consisted of a daily i.p. injection of $750 \mathrm{mg} / \mathrm{kg}$ in saline. Under these conditions, no side effects were observed. High-resolution ${ }^{1} \mathrm{H}$ MRS was performed on treated and control tumor extracts. A dual-phase extraction method based on methanol/chloroform/water was used to obtain lipid and water-soluble fractions from the tumors. We also investigated effects on metastases and ascites formation. Pantethine treatment resulted in slower tumor progression, decreased levels of phosphocholine and phosphatidylcholine, and reduced metastases and ascites occurrence. In conclusion, pantethine represents a novel potential, well-tolerated, therapeutic tool in patients with ovarian cancer. Further in vivo preclinical studies are needed to confirm the beneficial role of pantethine and to better understand its mechanism of action.

Keywords: choline metabolism, pantethine, ovarian cancer, orthotopic model, ascites, metastasis, high-resolution MRS

Abbreviations: FAS, fatty acid synthase; IHC, immunohistochemistry; MRI, magnetic resonance imaging; MRS, magnetic resonance spectroscopy; PC, phosphocholine; $\mathrm{PtCh}$, phosphatidylcholine. 


\section{INTRODUCTION}

Ovarian cancer is the leading cause of death from gynecological malignancies with an incidence of 220,000 cases worldwide per year (1). Although the prognosis in cases detected at an early stage is quite favorable, the vast majority of cases are diagnosed at an advanced stage, when 5-year survival rates are only $30-40 \%$. Median life expectancy for ovarian cancer patients is 5 years, and about $80 \%$ of diagnosed patients will eventually succumb to it (2). The poor prognosis of epithelial ovarian cancer (EOC) is due to a combination of the aggressive characteristics of the disease and an unpredictable response to front-line therapy, further compounded by late detection of the disease and resistance of ovarian cancers to current treatments (3). The primary treatment for EOC consists of aggressive cytoreductive surgery, followed by chemotherapy with platinum and taxane (4). Although platinum and taxane combination remains the standard treatment for EOC, new drug combinations (5) as well as different administration schedules (6) are being tested and might be reasonable options for first-line treatment of women with advanced EOC. Recently, the introduction of antiangiogenic drug combined to front-line treatment has been also proposed $(7,8)$. Current first-line chemotherapies for advanced diseases are listed in Table 1.

Metastases and malignant ascites are complications frequently observed in late-stage ovarian cancer. Intraperitoneal seeding is the most common route of dissemination (9), although direct invasion or dissemination through the lymphatics and vasculature also occur. Malignant ascites function as a permissive reactive tumor-host microenvironment and provides sustenance for floating tumor cells (10). This results in an abnormal build-up of fluid in the abdomen, causing discomfort, pain, problems with mobility and breathing, and other symptoms that decrease the quality of life. Despite the improvement of surgical approaches and drug development, EOC patients have experienced little improvement in overall survival in the last 30 years (11). New therapeutic strategies exploiting novel targets are urgently needed to minimize morbidity, improve survival rates, and to eventually cure patients.

In the present study, we applied magnetic resonance imaging (MRI) and high-resolution magnetic resonance spectroscopy (MRS) to assess the use of pantethine as a therapeutic agent in an orthotopic model of ovarian cancer. We used an orthotopic model in which the relevant tumor physiological environment is maintained and that frequently forms metastases and

TABLE 1 | Front-line treatment for ovarian cancer patients.

Carboplatinum + paclitaxel (1)

Platinum-based chemotherapy + anti-mitotic chemotherapy

Carboplatinum and pegylated doxorubicin (5)

Carboplatinum + weekly paclitaxel (6)

Carboplatinum and taxol + bevacizumab $(7,8)$
Platinum-based chemotherapy + intercalating DNA chemotherapy

Platinum-based chemotherapy + anti-mitotic chemotherapy

Platinum-based chemotherapy + anti-mitotic chemotherapy + angiogenesis inhibitor malignant ascites. Pantethine is the stable disulfide form of pantetheine, the precursor of vitamin B5 (pantothenic acid). As a part of the active moiety of coenzyme A (CoA), it is a key regulator in lipid metabolism (12-14). Pantethine has the advantage of being an anti-inflammatory and hypolipidemic agent with very few side effects. Pantethine has been shown to prevent the perivascular inflammation and to protect mice against the cerebral syndrome associated with malaria (15); the protection was associated with a significantly lower level of circulating tumor necrosis factor (TNF)- $\alpha$ (15). TNF- $\alpha$ has been linked to multiple steps of tumorigenesis, including cellular transformation, promotion, survival, proliferation, invasion, angiogenesis, and metastasis (16). Pantethine has also been shown to inhibit CXCL12/CXCR4-induced cell transendothelial migration (17). With its anti-inflammatory and hypolipidemic properties, pantethine appeared to be a good novel candidate against ovarian cancer progression, metastases, and ascites formation.

\section{MATERIALS AND METHODS}

\section{Cell Line and Tumor Implantation}

NIH: OVCAR3 cells from American Type Culture Collection (ATCC, VA, USA) were used in the present study. OVCAR3 cells are human epithelial ovary adenocarcinoma cells originally isolated from a malignant effusion. Cells were cultured in RPMI 1640 (Sigma Chemical Co., St. Louis, MO, USA) with 10\% fetal bovine serum (Sigma Chemical Co., St. Louis, MO, USA). Tumor implantation was performed using 6- to 8-week-old severe combined immunodeficient (SCID) female mice. We used a two-step process for orthotopic tumor implantation (Supplementary Material). We first generated subcutaneous tumors by inoculating a cell suspension of $2 \times 10^{6}$ cells in $0.05 \mathrm{ml}$ of Hanks balanced salt solution in the flank of SCID female mice. Once the tumor reached a size of $100-200 \mathrm{~mm}^{3}$, it was excised and cut into small pieces under sterile conditions. Orthotopic implantation was then performed by surgically transplanting a piece of tumor tissue onto the ovaries of a separate group of SCID mice. All surgical procedures and animal handling were performed in accordance with protocols approved by the Johns Hopkins University Institutional Animal Care and Use Committee and conformed to the Guide for the Care and Use of Laboratory Animals published by the NIH.

\section{Treatment Protocol}

The treatment was started when the tumors reached about $100 \mathrm{~mm}^{3}$ with a daily i.p. injection of saline for the control group, and pantethine (Sigma Chemical Co., St. Louis, MO, USA) diluted in saline for the treated group $(750 \mathrm{mg} / \mathrm{kg})$.

\section{In Vivo MR Examination}

Non-invasive MRI was used to assess tumor growth in deepseated tissue using $\mathrm{T}_{1}$-weighted imaging and diffusion-weighted imaging. All imaging studies were performed on a 4.7-T BrukerAvance (Bruker, Billerica, MA, USA) spectrometer 
using a home-built volume coil placed around the torso of the anesthetized mice. Animals were anesthetized with a mixture of ketamine $(6.25 \mathrm{mg} / \mathrm{kg})$ and acepromazine $(62.5 \mathrm{mg} / \mathrm{kg})$ administered i.p. A pad circulated with warm water was used to maintain animal body temperature. Multi-slice $\mathrm{T}_{1}$-weighted images and multi-slice diffusion-weighted images, with an inplane spatial resolution of $250 \mu \mathrm{m} \times 250 \mu \mathrm{m}(128 \times 128$ matrix, $32 \mathrm{~mm}$ field of view, $b$-value of $100 \mathrm{mT} / \mathrm{m}$ ), were acquired to localize the orthotopic tumors that appear hyperintense on these images.

\section{MR Spectroscopy of Dual Phase Extracts}

High-resolution proton MRS of tumor tissue extracts was applied to assess water phase and lipid phase metabolites in tumor extracts. Lipid- and water-soluble fractions were obtained from tumors using a dual-phase extraction method with methanol/chloroform/water (1/1/1) (18). Briefly, tissues were freeze-clamped and ground to powder. Ice-cold methanol was added to the powder, and the samples were homogenized. Ice-cold chloroform, followed by ice-cold water, was added, and the samples were kept at $4^{\circ} \mathrm{C}$ overnight for phase separation. Samples were centrifuged for $30 \mathrm{~min}$ at $15,000 \mathrm{~g}$ at $4^{\circ} \mathrm{C}$ to separate the phases. The water/methanol phase containing the water-soluble metabolites was treated with chelex (Sigma Chemical Co., St. Louis, MO, USA) for $10 \mathrm{~min}$ on ice to remove divalent cations. Methanol was removed by rotary evaporation, and the remaining water phase was lyophilized and stored at $-20^{\circ} \mathrm{C}$. The chloroform phase containing the lipids was dried in a stream of $\mathrm{N}_{2}$ and stored at $-20^{\circ} \mathrm{C}$. Water-soluble samples were dissolved in $0.5 \mathrm{ml}$ of $\mathrm{D}_{2} \mathrm{O}$ (Sigma Chemical Co., St. Louis, MO, USA) containing 3-(trimethylsilyl) propionic-2,2,3,3,-d4 acid (Sigma Chemical Co., St. Louis, MO, USA) as an internal concentration standard (sample $\mathrm{pH}$ of 7.4). Lipid samples were dissolved in $0.6 \mathrm{ml}$ of $\mathrm{CDCl}_{3} / \mathrm{CD}_{3} \mathrm{OD}(2 / 1)$ containing tetramethylsilane as an internal concentration standard $\left(\mathrm{CDCl}_{3}\right.$ and $\mathrm{CD}_{3} \mathrm{OD}$ premixed with tetramethylsilane by the manufacturer, Cambridge Isotope Laboratories, Inc.). Fully relaxed ${ }^{1} \mathrm{H}$ MR spectra of the extracts were acquired on a BrukerAvance 500 spectrometer operating at $11.7 \mathrm{~T}$ (BrukerBioSpin Corp., Billerica, MA, USA) using a 5-mm HX inverse probe and the following acquisition parameters: $30^{\circ}$ flip angle, $6,000 \mathrm{~Hz}$ sweep width, $12.7 \mathrm{~s}$ repetition time, time-domain data points of $32 \mathrm{k}$, and 128 transients (18). Spectra were analyzed using the Bruker XWIN-NMR 3.5 software (BrukerBioSpin). Integrals of the metabolites of interest were determined and normalized to the tumor weight. To determine concentrations, peak integration from ${ }^{1} \mathrm{H}$ spectra for all metabolites studied was compared to the internal standard.

\section{Metastases and Ascites}

Presence of ascites was recorded at necropsy. Lymph nodes, lungs, and livers were fixed in formalin, paraffin embedded, sectioned, and stained with hematoxylin and eosin (H\&E) for further analysis. The presence of metastases was checked on H\&E stained sections of the lymph nodes, liver, and lungs.

\section{Immunohistochemistry}

The $5-\mu \mathrm{m}$ thick formalin fixed sections were used for Immunohistochemistry (IHC) analysis. Antigen retrieval was achieved by boiling sections in citrate buffer solution $(\mathrm{pH} 6)$ for $20 \mathrm{~min}$. Sections were stained for proliferation using Ki-67 (rabbit polyclonal, Thermo Fisher, Rockford, IL, USA, 1:100 dilution), and for apoptosis using Caspase-3 (8G10, rabbit polyclonal, Cell Signaling, Danvers, MA, USA, 1:100 dilution) following standard protocols, and further processed by addition of biotinylated anti-rabbit IgG and ABC reagent (PK-4001, Vector laboratories, Burlingame, CA, USA). Detection was achieved by addition of the chromogen DAB (3, 3'-diaminobenzidine, Dako, Carpinteria, CA, USA). Images were captured by scanning the immunostained sections at high resolution on an Aperio ScanScope ${ }^{\circledR}$ CS System at $20 \times$ resolution (Leica Biosystems Inc., Buffalo Grove, IL, USA). Analysis of the slides was performed using the algorithms and protocols developed by the company.

\section{Toxicity Analysis}

The toxicity analyses were performed in MDA-MB-231 tumorbearing mice. The $2 \times 10^{6}$ cells were injected orthotopically into the mammary fat pad of 6 - to 8 -week-old female SCID mice. The treatment was started when the tumors reached about $100 \mathrm{~mm}^{3}$ with a daily i.p. injection of saline for the control group and pantethine for the treated group $(750 \mathrm{mg} / \mathrm{kg})(n=5)$. Mice were treated for 3 weeks and weighed once a week. At the end of the treatment period, mice were sacrificed. Kidney and liver function were evaluated from serum creatinine, blood urea nitrogen (BUN), serum alanine aminotransferase (ALT), and aspartate aminotransferase (AST) levels obtained at the Johns Hopkins University School of Medicine Phenotyping Core Facility, using spectrophotometric measurements obtained with an automated Vet Ace Clinical Chemistry system (Alfa Wasserman Diagnostic Technologies LLC, NJ, USA).

\section{Statistical Analysis}

Values were displayed as mean \pm SEM. Statistical significance was evaluated using the Student's $t$-test; $p<0.05$ was considered significant.

\section{RESULTS}

To assess the efficacy of pantethine on tumor progression, metastases, and ascites formation, we used an orthotopic model of ovarian cancer. The orthotopic implantation was performed to maintain the relevant tumor physiological environment. Ovarian cancer cells are typically injected into the peritoneal cavity, inducing ascites and peritoneal spread of tumor, but most cell lines do not form solid tumors. Instead, here we performed microsurgical orthotopic implantation of ovarian cancer tissue onto the ovary of female SCID mice. In our model, ascites and metastases in the peritoneal cavity, in the liver, on the diaphragm, and in distal lymph nodes are frequent, similar to human disease. Tumor growth was measured following implantation by imaging the mice weekly with MRI (Figure 1). The treatment consisting of daily i.p. injections of pantethine at a dose of $750 \mathrm{mg} / \mathrm{kg}$ 


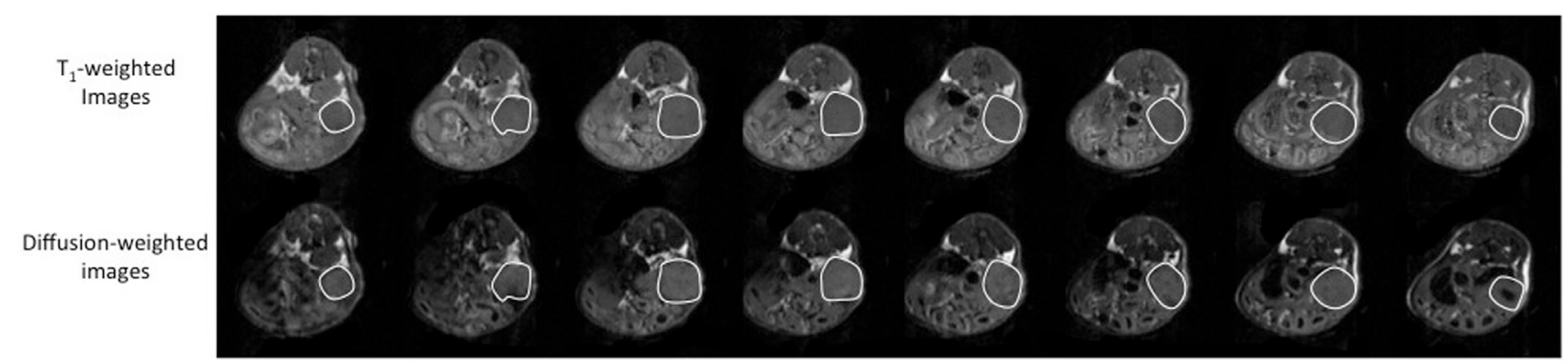

FIGURE 1 | Representative adjacent $\mathrm{T}_{1}$-weighted images (top row) and diffusion-weighted images (bottom row) of an orthotopically implanted OVCAR3 tumor-bearing mouse. The tumor is highlighted by a white line. The tumor volume was measured by determining the tumor area on each 1-mm thick slice and by adding the areas to calculate the total tumor volume.

commenced when tumors were $\sim 100 \mathrm{~mm}^{3}$. Under these conditions, no side effects and no significant weight loss were observed in the treated group compared to the control group $(20.8 \pm 0.9$ versus $22.2 \pm 2.8 \mathrm{~g}$, respectively). The control group was injected daily with saline. Tumor growth was followed weekly noninvasively by MRI on a 4.7-T spectrometer. Tumor areas were measured for each 1-mm thick slice, and the values were added to assess the total tumor volume. We observed a significant reduction of tumor growth in the treated group compared to the control group (Figure 2).

When we sacrificed the mice after 4 weeks of treatment, we observed liver metastases in $86 \%$ of control mice (6/7), but only in $43 \%$ of treated mice (2/7), lungs metastases in $29 \%$ of control mice $(2 / 7)$, and none in treated mice and ascites in $86 \%$ of control mice (6/7), and $29 \%$ of treated mice (2/7) (Figure 3). IHC analysis of tumor sections did not show any statistically significant differences in proliferation rates (Figures 4A,C). Higher levels of caspase- 3 were measured in the treated tumors compared to control tumors (Figures $4 \mathrm{~B}, \mathrm{C}$ ).

To assess the effect of the treatment on the tumor metabolism, we analyzed tumor extracts with high-resolution ${ }^{1} \mathrm{H}$ MRS. We performed dual-phase extraction of the tumors to assess the lipid phase and the water phase. Representative water phase ${ }^{1} \mathrm{H} \mathrm{MR}$ spectra centered around the $3.2 \mathrm{ppm}$ region of a control tumor and a pantethine-treated tumor are shown in Figures $\mathbf{5 A , B}$, respectively. A significant decrease of phosphocholine (PC) in the treated tumors was observed (Figure 5C). No differences were observed in the other metabolites measured, including lactate.

We next measured the lipid concentration in the lipid phase. Representative spectra of a control tumor and a treated tumor are shown in Figures 6A,B, respectively. Analysis of the spectra revealed a significant decrease of phosphatidylcholine (PtCho) in the pantethine-treated tumors compared to the controls (Figure 6C). No differences were observed in the other lipids assessed (Figures 6C,D).

Renal and hepatic cytotoxicity studies were conducted in MDA-MB-231 tumor-bearing mice. We did not observe any weight loss following 3 weeks of pantethine treatment (Figure 7A). Blood analysis performed at the end of the treatment

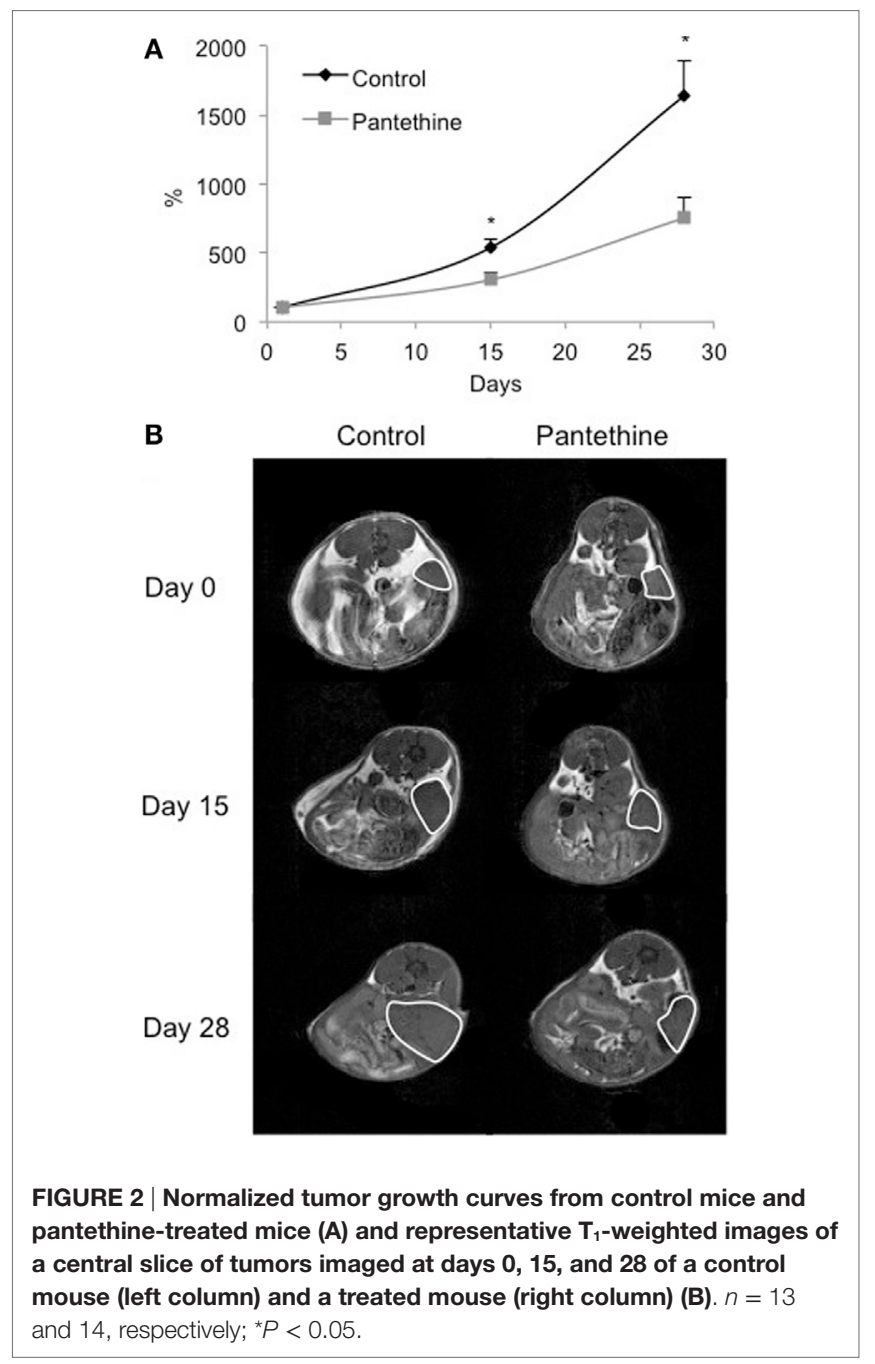

period revealed neither hepatic (Figure 7B) nor renal toxicity (Figure 7C), as shown by the absence of significant differences in the levels of BUN, creatinine, AST, and ALT between the control mice and the pantethine-treated mice. 

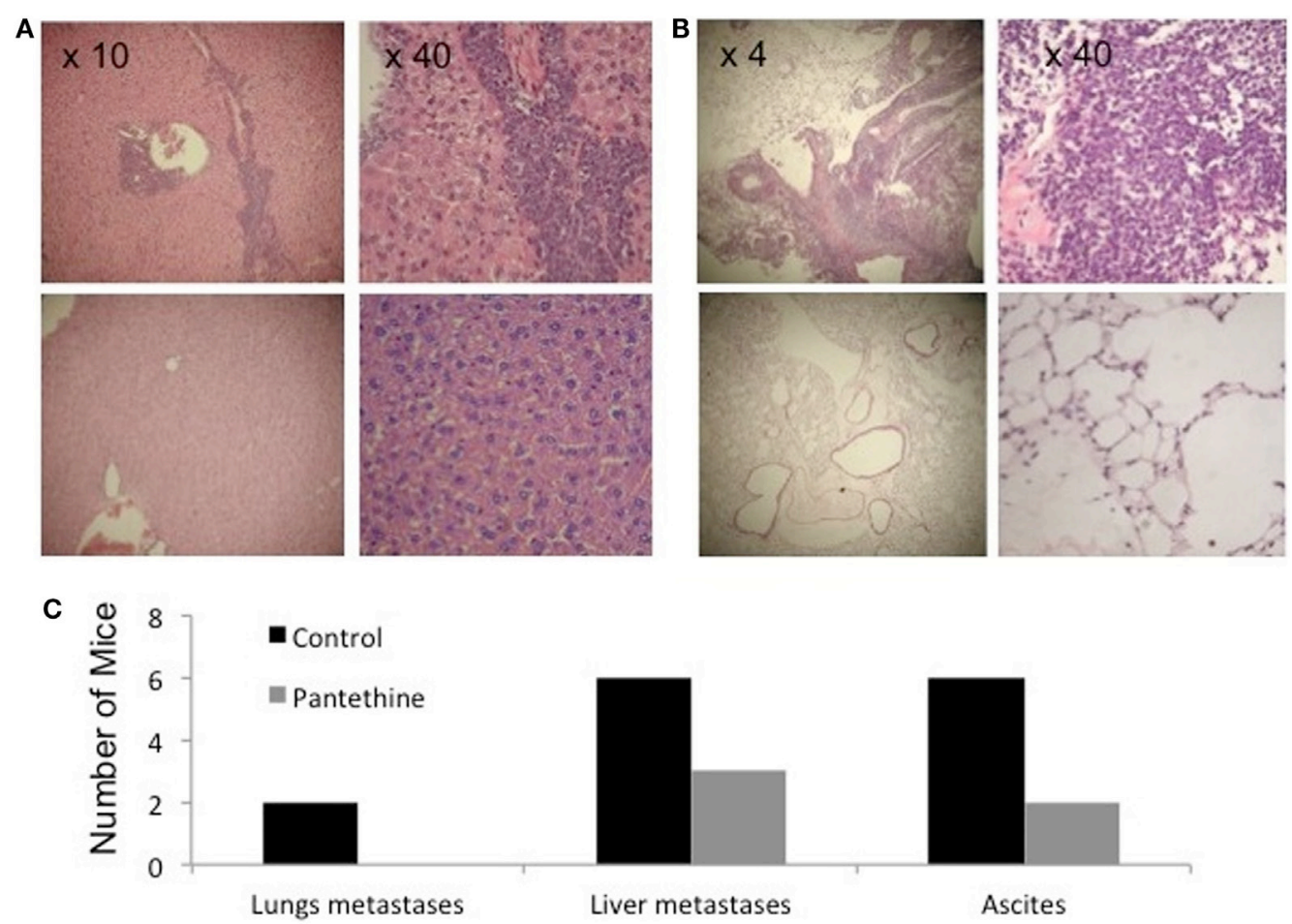

FIGURE 3 | Representative H\&E stained sections of liver (A) and lungs (B) from a control mouse (top row) and a treated mouse (bottom row). (C) Histogram representing the number of control and treated mice with metastases in the lungs, in the liver, and with ascites $(n=7)$.

A

Ki67
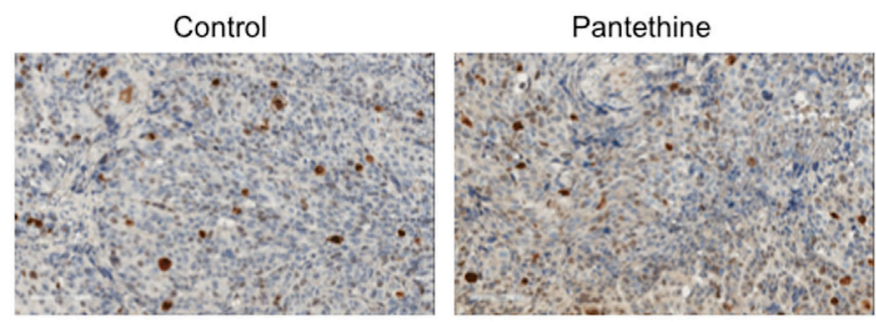

B
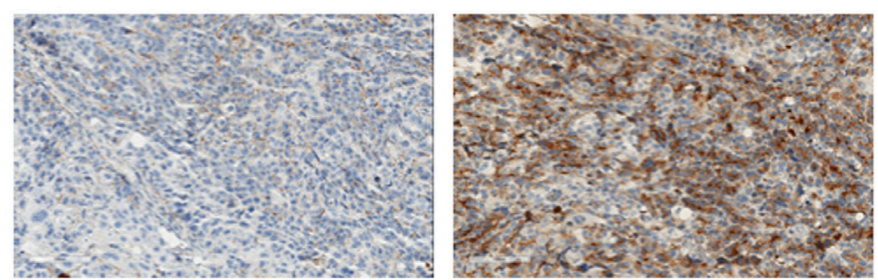

C

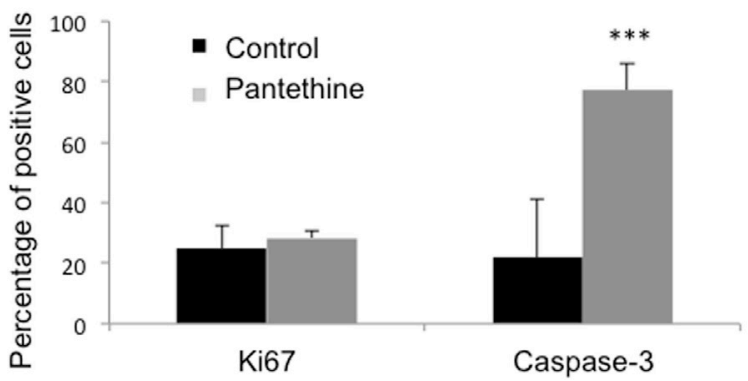

FIGURE 4 | Representative IHC stained sections of control and treated tumors for Ki-67 (A) and Caspase-3 (B). (C) Histogram representing the percentage of positive cells for each marker in control and treated tumors $\left(n=3\right.$; mean $\pm S D$ are represented; $\left.{ }^{\star \star \star} P<0.001\right)$. 


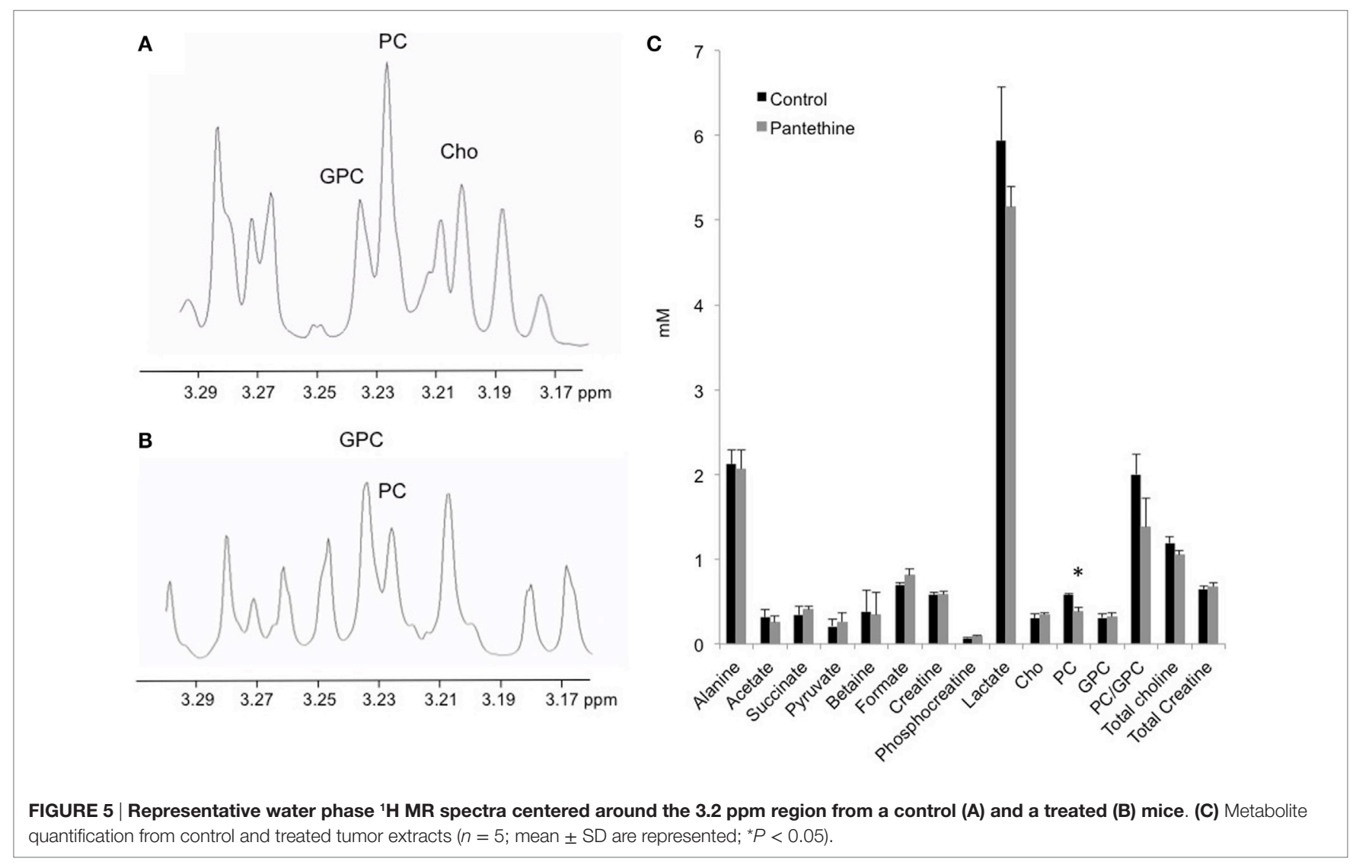

FIGURE 5 | Representative water phase ${ }^{1} \mathrm{H}$ MR spectra centered around the $3.2 \mathrm{ppm}$ region from a control (A) and a treated (B) mice. (C) Metabolite ion from control and treated tumor extracts $(n=5$; mean $+\mathrm{SD}$ are represented. * $P<0.05)$
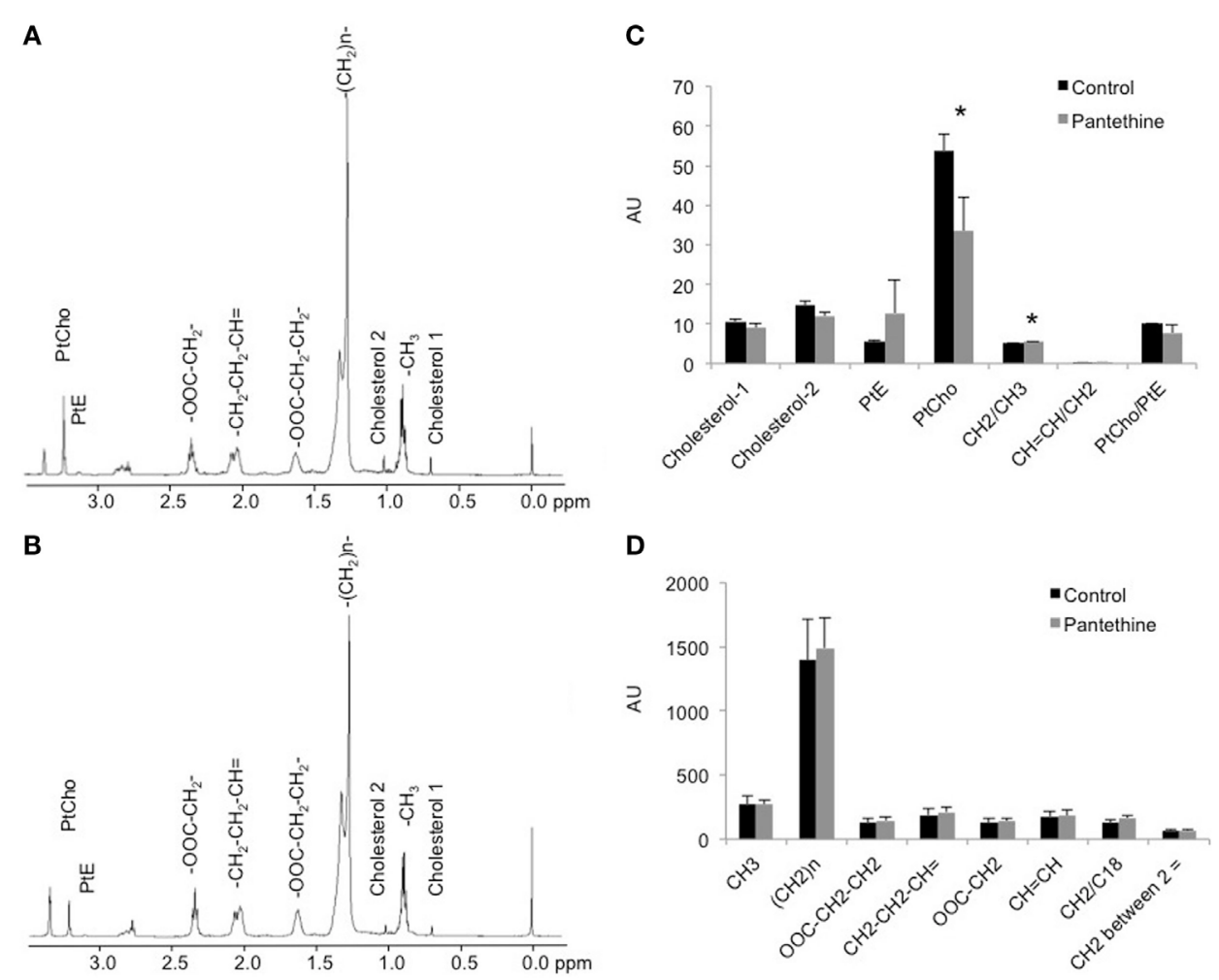

FIGURE 6 | Representative lipid phase ${ }^{1} \mathrm{H}$ MR spectra from a control (A) and a treated (B) mice. (C,D) Lipid quantification in arbitrary unit (AU) from control and treated tumor extracts $\left(n=5 ;{ }^{*} P<0.05\right)$. 


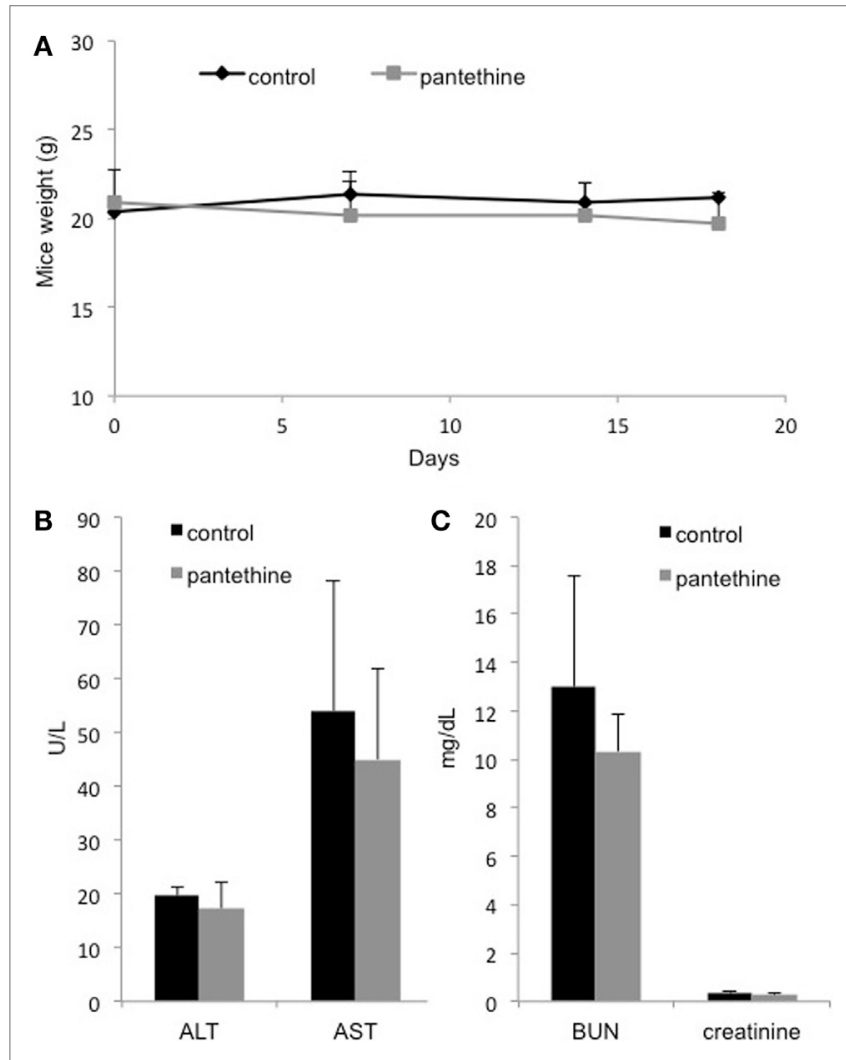

FIGURE 7 | (A) Weight curves from control and treated mice. Serum levels of ALT, AST, (B) BUN, and creatinine (C) in treated and control mice. $n=5$; mean \pm SD are presented.

\section{DISCUSSION}

The majority of ovarian cancer studies in mouse models use xenografts that are obtained either after subcutaneous implantation (19) or intraperitoneal injection of cancer cells (20). To mimic the tumor microenvironment, more recent models involved direct injection of cells into the ovarian intrabursa $(21,22)$. Another technique involving the implantation of a preparation of tumor solid pellets into the ovarian bursa was recently described (23). In this model, a pellet was prepared by embedding tumor cells into a collagen matrix to control the number of cells, and to limit their leakage during the injection (23). In the present study, we engrafted a piece of tumor tissue onto the ovary to avoid spilling of cancer cells and to maintain the tumor tissue microenvironment. We have used this technique in the past for prostate cancer (24) and for pancreatic cancer (25). In our model, ascites and metastases in the peritoneal cavity, in the liver, on the diaphragm, and in distal lymph nodes are frequent, similar to human disease, since ovarian carcinoma usually metastasizes along the peritoneum throughout the pelvic and abdominal cavity. In ovarian cancer patients, metastases can be found in lung, skin, pleura, mediastinal, and lymph nodes, and also in bone, brain, or gastrointestinal track (26).

Using our orthotopic model, we identified pantethine as a promising new drug against ovarian cancer, targeting not only tumor progression but also metastases occurrence, and ascites formation. The orthotopic tumor growth could be followed noninvasively using MRI, and we observed slower tumor progression in the treated mice compared to the non-treated ones. While there were no differences in cell proliferation, increased caspase- 3 was observed in the treated tumors, linking the tumor growth reduction to an increase in apoptosis. High-resolution ${ }^{1} \mathrm{H}$ MRS analysis of tumor extracts revealed a significant decrease of PC and PtCho concentrations in the tumors from treated mice compared to the untreated controls. We used a high dose of pantethine and did not observe any side effects. Pantethine is rapidly eliminated into the urine allowing its administration in humans at a reasonable dose using a slow dispensing device.

Abnormal choline metabolism continues to be identified as one of the most consistent hallmarks of cancer (27). The molecular causes are being gradually unraveled and are providing potential novel targets in the treatment of cancer. Iorio et al. demonstrated that EOC possessed an altered MRS-choline profile, characterized by increased PC content (28). Several studies have demonstrated that targeting choline kinase resulted in a decrease of PC and a reduction of tumor growth $(29,30)$. Here, we observed a reduction of tumor progression that was associated with a decrease of $\mathrm{PC}$ and PtCho.

Several known properties of pantethine may explain these results. A previous study described the inhibition of PtCho synthesis in vitro in rat liver microsomal preparations with pantetheine and CoA (31). Here, we observed an in vivo effect of pantethine on PtCho level in orthotopically implanted OVCAR3 tumor. Pantethine inhibited fatty acid synthase (FAS), as demonstrated in isolated rat hepatocytes by Bocos and Herrera (32). FAS synthesizes fatty acids using $4^{\prime}$-phospho-pantetheine, which acts as a universal mechanism of transport of intermediates (33-35). Pantethine may inhibit FAS activity through the alteration of the thiol group of the $4^{\prime}$ - phospho-pantetheine arm, which covalently carries the pathway intermediates. High FAS activity has been observed in most ovarian cancers and is strongly associated with high aggressiveness and poor patient survival. Inhibition of FAS activity has been shown to be cytotoxic to human cancer cells in vitro and in vivo (36). Pantethine not only affects cellular fatty acid metabolism but also displays anti-inflammatory properties by maintaining the asymmetric distribution of cell membrane phosphatidylserine, resulting in the inhibition of cellular response to proinflammatory factors (15).

It was recently shown that pantethine affects lipid raft composition causing a decline of the proportion of saturated fatty acid, an increase in mono- and polyunsaturated fatty acid, and a decrease of cholesterol content (17). These changes in raft composition led to an impairment of CXCL12 to bind to its target (17). The CXCL12-CXCR4 axis promotes proliferation, migration, invasion, and metastasis in ovarian cancer (37), therefore its alteration by pantethine may be a mechanism for the reduction of metastases observed in our study.

Finally, it was recently shown that obesity contributes to ovarian cancer metastases formation (38). Adipose tissue is a key component of the ovarian cancer metastatic microenvironment $(39,40)$. Increased body fat enhances tumor cell-mesothelial cell adhesion and promotes intraperitoneal metastatic dissemination (38). 
As a known hypolipidemic agent, pantethine may influence metastases formation through its hypolipidemic effects.

In conclusion, pantethine represents a novel potential therapeutic option in patients with ovarian cancer, since it is a wellknown and well-tolerated molecule. Further in vivo preclinical studies are needed to confirm the beneficial role of pantethine in ovarian cancer and to better understand its mechanism of action.

\section{AUTHOR CONTRIBUTIONS}

MP: conception and design of the work; acquisition, analysis, and interpretation of data; drafting the work; final approval of the version to be published; and agreement to be accountable for all aspects of the work. BK, FW, and YM: acquisition, analysis, and interpretation of data; final approval of the version to be

\section{REFERENCES}

1. Jayson GC, Kohn EC, Kitchener HC, Ledermann JA. Ovarian cancer. Lancet (2014) 384(9951):1376-88. doi:10.1016/S0140-6736(13)62146-7

2. Schmidt C. Ovarian cancer treatments on the horizon. J Natl Cancer Inst (2011) 103(17):1284-5. doi:10.1093/jnci/djr343

3. Bast RC Jr. Molecular approaches to personalizing management of ovarian cancer. Ann Oncol (2011) 22(Suppl 8):viii5-15. doi:10.1093/annonc/mdr516

4. Krivak TC, Lele S, Richard S, Secord AA, Leath CA III, Brower SL, et al. A chemoresponse assay for prediction of platinum resistance in primary ovarian cancer. Am J Obstet Gynecol (2014) 211(1):e61-8. doi:10.1016/j. ajog.2014.02.009

5. Pignata S, Scambia G, Ferrandina G, Savarese A, Sorio R, Breda E, et al. Carboplatin plus paclitaxel versus carboplatin plus pegylated liposomal doxorubicin as first-line treatment for patients with ovarian cancer: the MITO-2 randomized phase III trial. J Clin Oncol (2011) 29(27):3628-35. doi:10.1200/ JCO.2010.33.8566

6. Pignata S, Scambia G, Katsaros D, Gallo C, Pujade-Lauraine E, De Placido $S$, et al. Carboplatin plus paclitaxel once a week versus every 3 weeks in patients with advanced ovarian cancer (MITO-7): a randomised, multicentre, open-label, phase 3 trial. Lancet Oncol (2014) 15(4):396-405. doi:10.1016/ S1470-2045(14)70049-X

7. Burger RA, Brady MF, Bookman MA, Fleming GF, Monk BJ, Huang H, et al. Incorporation of bevacizumab in the primary treatment of ovarian cancer. $N$ Engl J Med (2011) 365(26):2473-83. doi:10.1056/NEJMoa1104390

8. Perren TJ, Swart AM, Pfisterer J, Ledermann JA, Pujade-Lauraine E, Kristensen $\mathrm{G}$, et al. A phase 3 trial of bevacizumab in ovarian cancer. N Engl J Med (2011) 365(26):2484-96. doi:10.1056/NEJMoa1103799

9. Kyriazi S, Kaye SB, Desouza NM. Imaging ovarian cancer and peritoneal metastases-current and emerging techniques. Nat Rev Clin Oncol (2010) 7(7):381-93. doi:10.1038/nrclinonc.2010.47

10. Said N, Socha MJ, Olearczyk JJ, Elmarakby AA, Imig JD, Motamed K. Normalization of the ovarian cancer microenvironment by SPARC. Mol Cancer Res (2007) 5(10):1015-30. doi:10.1158/1541-7786.MCR-07-0001

11. Vaughan S, Coward JI, Bast RC Jr, Berchuck A, Berek JS, Brenton JD, et al. Rethinking ovarian cancer: recommendations for improving outcomes. Nat Rev Cancer (2011) 11(10):719-25. doi:10.1038/nrc3144

12. Branca D, Scutari G, Siliprandi N. Pantethine and pantothenate effect on the CoA content of rat liver. Int J Vitam Nutr Res (1984) 54(2-3):211-6.

13. Wittwer CT, Graves CP, Peterson MA, Jorgensen E, Wilson DE, Thoene JG, et al. Pantethine lipomodulation: evidence for cysteamine mediation in vitro and in vivo. Atherosclerosis (1987) 68(1-2):41-9. doi:10.1016/00219150(87)90092-X

14. Cighetti G, Del Puppo M, Paroni R, Galli Kienle M. Modulation of HMG-CoA reductase activity by pantetheine/pantethine. Biochim Biophys Acta (1988) 963(2):389-93. doi:10.1016/0005-2760(88)90306-2

15. Penet MF, Abou-Hamdan M, Coltel N, Cornille E, Grau GE, de Reggi M, et al. Protection against cerebral malaria by the low-molecular-weight thiol published; and agreement to be accountable for all aspects of the work. DM, FP, MD, BG, and ZB: design of the work; revising the work; final approval of the version to be published; and agreement to be accountable for all aspects of the work.

\section{FUNDING}

This work was supported by NIH P50CA103175, P30CA006973, The HERA Foundation, and The Tina's Wish Foundation.

\section{SUPPLEMENTARY MATERIAL}

The Supplementary Material for this article can be found online at http://journal.frontiersin.org/article/10.3389/fonc. 2016.00244/full\#supplementary-material.

pantethine. Proc Natl Acad Sci U S A (2008) 105(4):1321-6. doi:10.1073/ pnas.0706867105

16. Aggarwal BB, Shishodia S, Sandur SK, Pandey MK, Sethi G. Inflammation and cancer: how hot is the link? Biochem Pharmacol (2006) 72(11):1605-21. doi:10.1016/j.bcp.2006.06.029

17. van Gijsel-Bonnello M, Acar N, Molino Y, Bretillon L, Khrestchatisky M, de Reggi M, et al. Pantethine alters lipid composition and cholesterol content of membrane rafts, with down-regulation of CXCL12-induced T cell migration. J Cell Physiol (2015) 230(10):2415-25. doi:10.1002/jcp.24971

18. Glunde K, Raman V, Mori N, Bhujwalla ZM. RNA interference-mediated choline kinase suppression in breast cancer cells induces differentiation and reduces proliferation. Cancer Res (2005) 65(23):11034-43. doi:10.1158/00085472.CAN-05-1807

19. Pisanu ME, Ricci A, Paris L, Surrentino E, Liliac L, Bagnoli M, et al. Monitoring response to cytostatic cisplatin in a HER2(+) ovary cancer model by MRI and in vitro and in vivo MR spectroscopy. Br J Cancer (2014) 110(3):625-35. doi:10.1038/bjc.2013.758

20. Canese R, Pisanu ME, Mezzanzanica D, Ricci A, Paris L, Bagnoli M, et al. Characterisation of in vivo ovarian cancer models by quantitative $1 \mathrm{H}$ magnetic resonance spectroscopy and diffusion-weighted imaging. NMR Biomed (2012) 25(4):632-42. doi:10.1002/nbm.1779

21. Cordero $A B$, Kwon $Y$, Hua $X$, Godwin $A K$. In vivo imaging and therapeutic treatments in an orthotopic mouse model of ovarian cancer. J Vis Exp (2010) (42):e2125. doi:10.3791/2125

22. Zhang H, Gao X, Yang Y, Wang W, Liu J, Liang Y, et al. New construction of an animal model for the orthotopic transplantation of an ovarian tumor. J Ovarian Res (2014) 7:64. doi:10.1186/1757-2215-7-64

23. Xu X, Ayub B, Liu Z, Serna VA, Qiang W, Liu Y, et al. Anti-miR182 reduces ovarian cancer burden, invasion, and metastasis: an in vivo study in orthotopic xenografts of nude mice. Mol Cancer Ther (2014) 13(7):1729-39. doi:10.1158/1535-7163.MCT-13-0982

24. Penet MF, Pathak AP, Raman V, Ballesteros P, Artemov D, Bhujwalla ZM. Noninvasive multiparametric imaging of metastasis-permissive microenvironments in a human prostate cancer xenograft. Cancer Res (2009) 69(22):8822-9. doi:10.1158/0008-5472.CAN-09-1782

25. Penet MF, Shah T, Bharti S, Krishnamachary B, Artemov D, Mironchik Y, et al. Metabolic imaging of pancreatic ductal adenocarcinoma detects altered choline metabolism. Clin Cancer Res (2015) 21(2):386-95. doi:10.1158/10780432.CCR-14-0964

26. Thibault B, Castells M, Delord JP, Couderc B. Ovarian cancer microenvironment: implications for cancer dissemination and chemoresistance acquisition. Cancer Metastasis Rev (2013) 33(1):17-39. doi:10.1007/s10555-013-9456-2

27. Glunde K, Penet MF, Jiang L, Jacobs MA, Bhujwalla ZM. Choline metabolism-based molecular diagnosis of cancer: an update. Expert Rev Mol Diagn (2015) 15(6):735-47. doi:10.1586/14737159.2015.1039515

28. Iorio E, Mezzanzanica D, Alberti P, Spadaro F, Ramoni C, D’Ascenzo S, et al. Alterations of choline phospholipid metabolism in ovarian tumor progression. Cancer Res (2005) 65(20):9369-76. doi:10.1158/0008-5472.CAN-05-1146 
29. Krishnamachary B, Glunde K, Wildes F, Mori N, Takagi T, Raman V, et al. Noninvasive detection of lentiviral-mediated choline kinase targeting in a human breast cancer xenograft. Cancer Res (2009) 69(8):3464-71. doi:10.1158/ 0008-5472.CAN-08-4120

30. Granata A, Nicoletti R, Perego P, Iorio E, Krishnamachary B, Benigni F, et al. Global metabolic profile identifies choline kinase alpha as a key regulator of glutathione-dependent antioxidant cell defense in ovarian carcinoma. Oncotarget (2015) 6(13):11216-30. doi:10.18632/oncotarget. 3589

31. Sribney M, Knowles CL, Lyman EM. Regulation of phosphatidylcholine synthesis in rat liver endoplasmic reticulum. Biochem J (1976) 156(3):507-14. doi:10.1042/bj1560507

32. Bocos C, Herrera E. Pantethine stimulates lipolysis in adipose tissue and inhibits cholesterol and fatty acid synthesis in liver and intestinal mucosa in the normolipidemic rat. Environ Toxicol Pharmacol (1998) 6(1):59-66. doi:10.1016/S1382-6689(98)00020-9

33. Kleinkauf H, Von Dohren H. A nonribosomal system of peptide biosynthesis. Eur J Biochem (1996) 236(2):335-51. doi:10.1111/j.1432-1033.1996. 00335.x

34. Marahiel MA, Stachelhaus T, Mootz HD. Modular peptide synthetases involved in nonribosomal peptide synthesis. Chem Rev (1997) 97(7):2651-74. doi:10.1021/cr960029e

35. Quadri LE, Weinreb PH, Lei M, Nakano MM, Zuber P, Walsh CT. Characterization of Sfp, a Bacillus subtilis phosphopantetheinyl transferase for peptidyl carrier protein domains in peptide synthetases. Biochemistry (1998) 37(6):1585-95. doi:10.1021/bi9719861

36. Ross J, Najjar AM, Sankaranarayanapillai M, Tong WP, Kaluarachchi K, Ronen SM. Fatty acid synthase inhibition results in a magnetic resonance-detectable drop in phosphocholine. Mol Cancer Ther (2008) 7(8):2556-65. doi:10.1158/1535-7163.MCT-08-0015

37. Guo Q, Gao BL, Zhang XJ, Liu GC, Xu F, Fan QY, et al. CXCL12-CXCR4 axis promotes proliferation, migration, invasion, and metastasis of ovarian cancer. Oncol Res (2015) 22(5):247-58. doi:10.3727/096504015X14343704124430

38. Liu Y, Metzinger MN, Lewellen KA, Cripps SN, Carey KD, Harper EI, et al. Obesity contributes to ovarian cancer metastatic success through increased lipogenesis, enhanced vascularity, and decreased infiltration of M1 macrophages. Cancer Res (2015) 75(23):5046-57. doi:10.1158/0008-5472. CAN-15-0706

39. Lengyel E. Ovarian cancer development and metastasis. Am J Pathol (2010) 177(3):1053-64. doi:10.2353/ajpath.2010.100105

40. Nieman KM, Kenny HA, Penicka CV, Ladanyi A, Buell-Gutbrod R, Zillhardt $\mathrm{MR}$, et al. Adipocytes promote ovarian cancer metastasis and provide energy for rapid tumor growth. Nat Med (2011) 17(11):1498-503. doi:10.1038/ nm. 2492

Conflict of Interest Statement: The authors declare that the research was conducted in the absence of any commercial or financial relationships that could be construed as a potential conflict of interest.

Copyright (C) 2016 Penet, Krishnamachary, Wildes, Mironchik, Mezzanzanica, Podo, de Reggi, Gharib and Bhujwalla. This is an open-access article distributed under the terms of the Creative Commons Attribution License (CC BY). The use, distribution or reproduction in other forums is permitted, provided the original author(s) or licensor are credited and that the original publication in this journal is cited, in accordance with accepted academic practice. No use, distribution or reproduction is permitted which does not comply with these terms. 